\title{
IMPLEMENTATION OF ENDOVASCULAR THERAPIES FOR STROKE IS BOOSTING CERVICAL ARTERY DISSECTION DIAGNOSIS
}

Pérez-Sánchez $\mathrm{S}^{1}$, Domínguez-Mayoral A ${ }^{1}$, Gamero MA ${ }^{1}$, De Torres Chacón $\mathrm{R}^{1}$, Moniche $\mathrm{F}^{2}$, Escudero $\mathrm{I}^{2}$, Zapara-Arriaza $E^{3}$, Ortega J33, González $A^{3}$, Montaner J3

${ }^{1}$ Neurology Department. Virgen Macarena University Hospital, Seville, Spain. ${ }^{2}$ Neurology Department. Virgen del Rocío University Hospital, Seville, Spain. ${ }^{3}$ Radiology Department. Virgen del Rocío University Hospital, Seville, Spain

\section{Background and Aims}

Cervical arterial dissection (CAD) is involved in 1 to $2 \%$ of all strokes in the general population and due to advanced imaging techniques, the detection of CAD has been rising in recent years. The aim of this study is to explore whether wide implementation of endovascular treatment for ischemic stroke is having an impact in the diagnosis of CAD.

\section{Methods}

All patients with CAD diagnosed in two University Hospitals in Seville-Andalusia from January 2015 to December 2017 were included. We collected demographic data, clinical variables, and information on diagnosis time and imaging techniques used for the diagnosis. Implementation of 24h-365d mechanical thrombectomy started in the city on August 15th, 2016. We compared diagnosis rates of CAD performed before this date and after this date. The diagnostic rate was based on all strokes admitted to both hospitals during these periods.
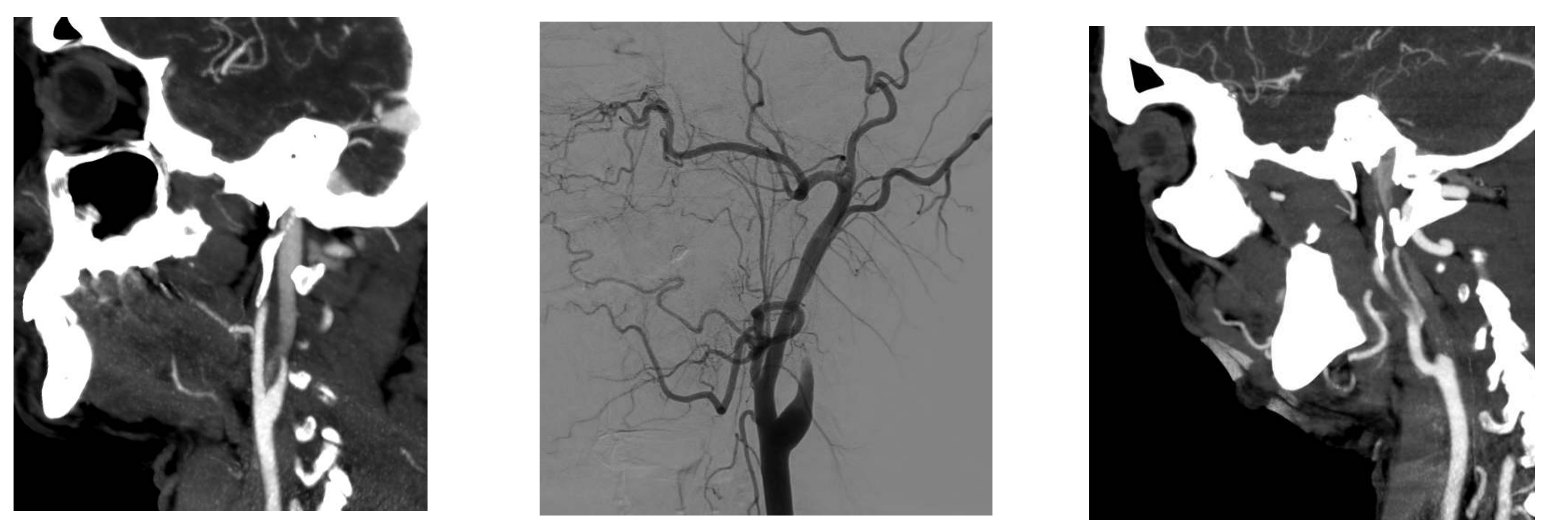

\section{Results}

We identified 41 CAD patients with a mean age of $47.34 \pm 11.55$ years. Baseline characteristics are shown in Table 1. In 17 cases, the diagnosis was done in the acute phase and angiography was made in all cases. Dissection was not suspected by CT-angiography in 11 cases due to small dissections (2) or total occlusion (9).

In the analysis of both periods, we found significantly more CAD patients diagnosed after the implementation of the endovascular treatment in the city $(p<0.001)$, and this finding was true for both hospitals. In fact, 13 patients were diagnosed in the before period $(1.10 \%$ of the total number of strokes in 19.5 months) and 28 in the period following endovascular therapy implementation $(2.19 \%$ of the total number of strokes in 16.5 months).

Table 1. Baseline Characteristics

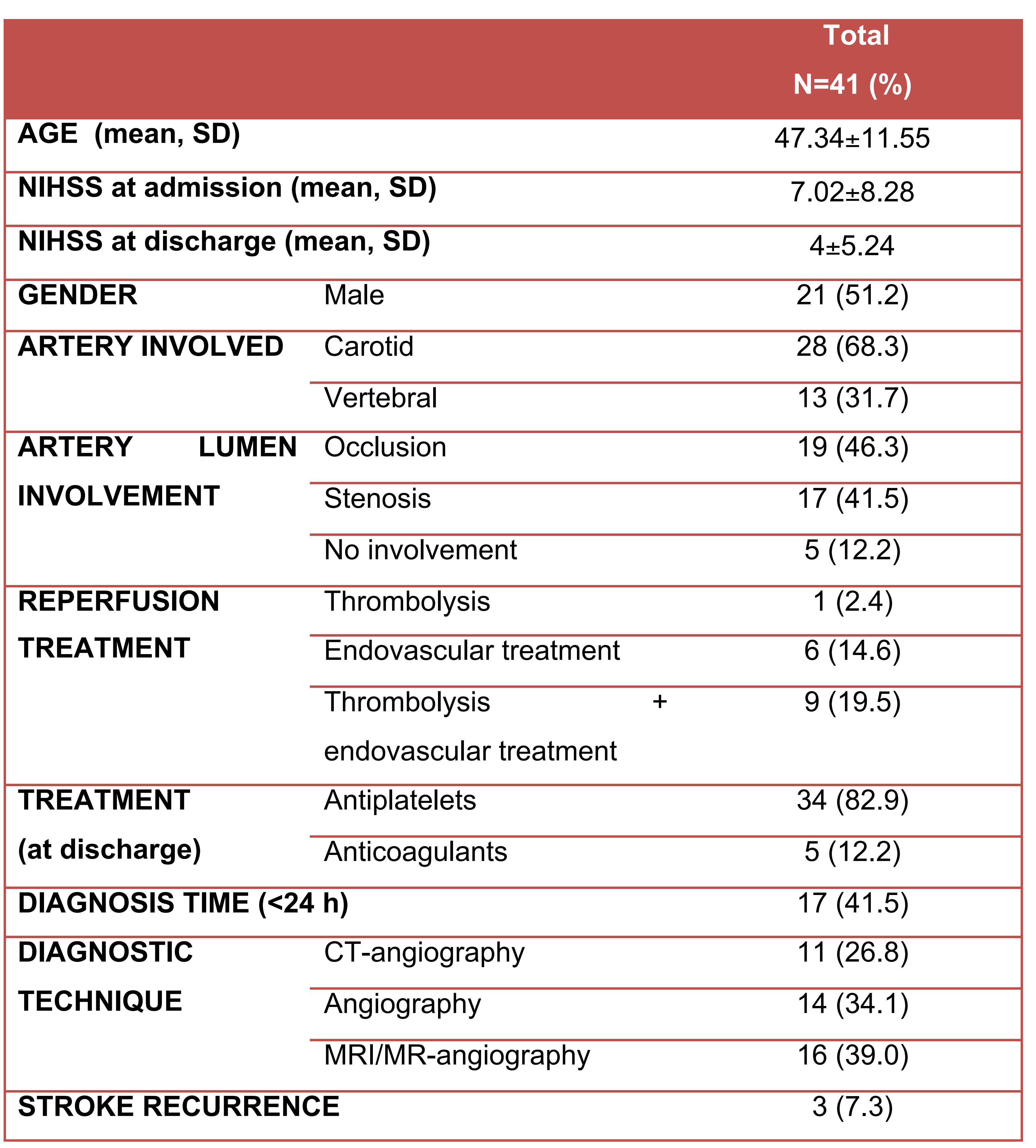

\section{Discussion}

CAD diagnosis has been rising in recent years, essentially due to the constant improving of imaging techniques. CTangiography might underestimate the incidence of CAD in many patients, and the rapid access to arteriography for thrombectomy is increasing this diagnosis, even in patients with a low level of dissection suspicion.

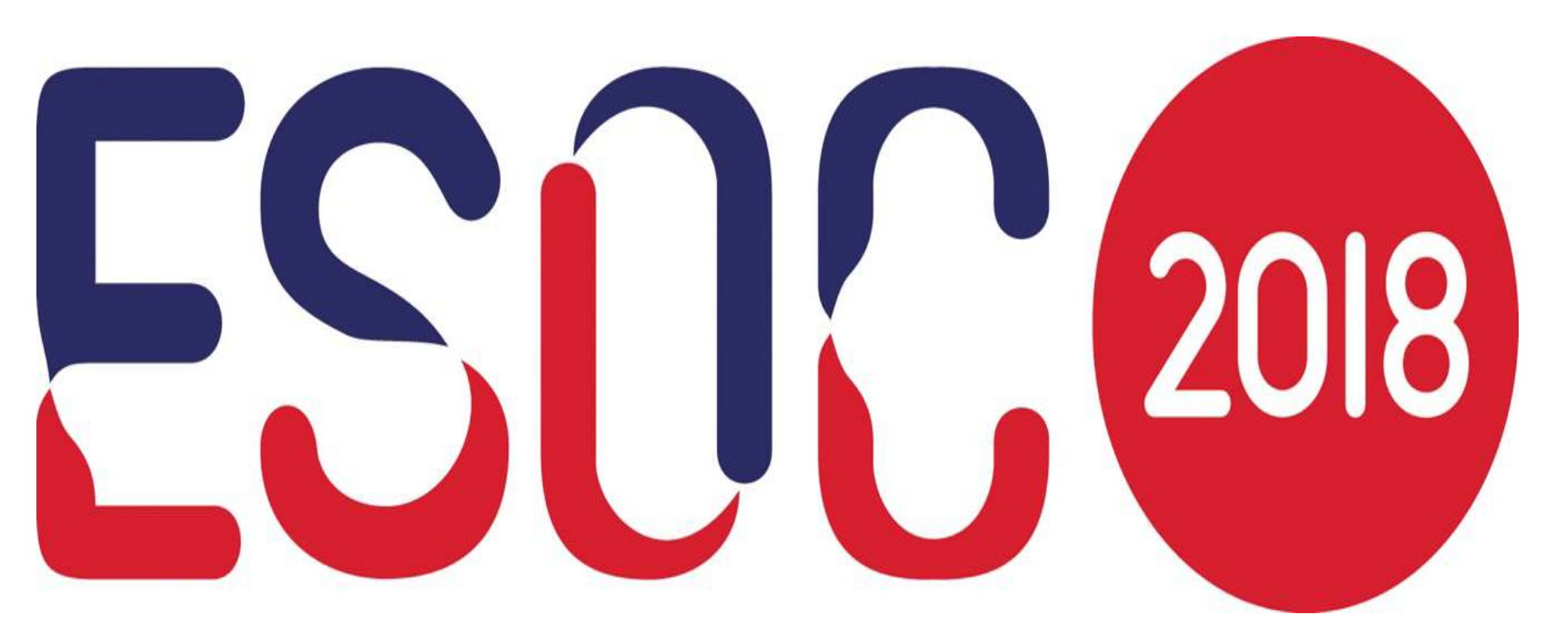

